\title{
SAFETY OF ANTIRETROVIRALS IN PREGNANCY
}

\author{
Polly Clayden
}

HIV i-Base, UK

\begin{abstract}
Highly active antiretroviral therapy (HAART) of three or more drugs is used extensively in industrialised countries for pregnant women with HIV, both to treat their own infection and to prevent mother-to-child transmission (MTCT). However, experts agree that current practice is based on limited randomised controlled trial data and guidelines are largely informed by observational studies and expert opinion. The British HIV Association (BHIVA) Pregnancy Guidelines note that 'The Cochrane Systematic Review of randomised controlled trials in this area shows how limited the guidelines would be were they to be restricted to such high-level evidence"1 (see box). Despite this, good results using HAART, showing both reduction in transmission to less than $2 \%$ and benefits to maternal health, have been reported from several cohorts and observational studies in industrialised countries. ${ }^{2-4}$

Research and programmes from resource-limited settings have largely focused on short-course regimens of antiretrovirals in pregnancy, with the objective of reducing MTCT, but data are gradually emerging from cohorts of women from these settings receiving HAART. ${ }^{5-7}$

That a woman should receive HAART if it is indicated for her own health is not controversial. Benefits to a woman's health clearly outweigh the known or theoretical risks associated with antiretroviral use, and her health and survival are paramount. The more complicated question is whether or not this is the best strategy for those women who do not yet meet the eligibility criteria for treatment but need intervention as prophylaxis to reduce transmission to their child, and it becomes still more difficult in settings where there may be limited monitoring of maternal and infant health. This article does not set out to discuss that question, but gives a brief overview of what is currently known about the risk/benefits associated with antiretroviral treatment, when it is considered appropriate, in pregnancy.
\end{abstract}

\section{MATERNAL TOXICITY}

Maternal antiretroviral toxicity appears to be largely similar to that reported in non-pregnant adults.

\section{NUCLEOSIDE REVERSE TRANSCRIPTASE INHIBITORS}

Nucleoside reverse transcriptase inhibitors (NRTIs) generally appear to be well tolerated in pregnancy and the

\section{The Cochrane Collaboration}

The Cochrane Collaboration performs systematic reviews of health care interventions (usually restricted to interventions demonstrated to be effective in randomised controlled trials) and disseminates the results. These reviews are the best single source of high-level evidence about the effects of health care.

The review of interventions to prevent MTCT of HIV found 18 trials eligible for inclusion (conducted in 16 countries and including 14398 participants). The
NRTIs zidovudine (AZT) and lamivudine (3TC) have been used most widely, with AZT being the only antiretroviral with an indication for use in pregnancy.

However, this class of drugs can cause adverse events associated with mitochondrial toxicity. In vitro the relative potency for inhibiting mitochondrial DNA polymerase is highest with didanosine (ddl), followed by stavudine (d4T), 3TC, AZT, abacavir (ABC), tenofovir (TDF) and emtricitabine (FTC).

In 2001 the US Food and Drug Administration (FDA) and

authors concluded that zidovudine (AZT) monotherapy, nevirapine monotherapy and caesarean section appear to be effective in decreasing the risk of transmission. In a 2007 update they added that a combination of AZT plus lamivudine appears to be effective.

The BHIVA guidelines state: 'This clearly does not reflect current practice but is extremely helpful in drawing attention to the lack of clinical trial data with HAART in pregnancy'.

http://www.cochrane.org/reviews/en/ab003510.html 
the European Medicines Agency (EMEA) warned that they had received reports of 3 fatal and 4 severe cases of lactic acidosis in women receiving $\mathrm{ddl}$ and $\mathrm{d} 4 \mathrm{~T}$ in pregnancy as part of triple combination therapy regimens. ${ }^{8}$ The use of these two drugs together in pregnancy is not recommended in most guidelines, and is restricted to women in need of treatment who have no other reasonable antiretroviral options.

\section{NEVIRAPINE}

Because efavirenz (EFV) is contraindicated in pregnancy (see below), the non-nucleoside reverse transcriptase inhibitor (NNRTI) nevirapine (NVP) has been the drug of this class most widely used in pregnancy.

In 2004, the originator manufacturer of NVP changed the Summary of Product Characteristics (SPC) to caution that women and patients with higher CD4 counts are at increased risk of hepatic toxicity when receiving NVP as part of a HAART regimen, particularly women with pretreatment CD4 counts $>250$ cells $/ \mu{ }^{9}{ }^{9}$

This change followed a retrospective review in which women with CD4 counts $>250$ cells/ $\mu$ l showed a 12 -fold higher risk of symptomatic hepatic adverse events compared with women with CD4 counts $<250$ cells/ $\mu$ l (11.0\% v. $0.9 \%$ ).

It is not clear whether the risk of hepatitis is the same for pregnant women, but before the SPC change a number of hepatitis-related deaths and major toxicities have been reported in pregnant women at higher CD4 cell counts receiving NVP. ${ }^{10}$

Conversely, in the Kisumu Breastfeeding Study in Kenya, in which AZT, 3TC and NVP were started at 34 weeks, $8.4 \%$ of mothers discontinued treatment due to hepatic toxicities but there was no difference between events among women above or below a CD4 count of 250 cells/ $\mu l{ }^{11}$ Furthermore, data from several large cohorts have not shown that pregnant women are at greater hepatoxicity risk. ${ }^{11-13}$

\section{PROTEASE INHIBITORS}

Protease inhibitors (PIs) are likely to become more widely prescribed in pregnancy in resource-limited settings as guidelines change to recommend earlier initiation of therapy at CD4 counts of $<350$ cells/ $\mu$ land universal treatment of pregnant women to prevent MTCT (as recommended in the Southern African Clinicians Society guidelines ${ }^{14}$ ) and because of their use in second-line regimens.

Higher than expected rates of hyperglycaemia and diabetes have been associated with PI use in pregnancy in some reports but not others.

Women receiving $\mathrm{PI}$-containing HAART have been shown to have a higher risk (3.5\%) of developing diabetes mellitus while pregnant compared with HIV-negative women. Compared with HIV-positive women receiving either
NRTIs only or no therapy, the risk also increased (1.35\%) $(p=0.025) .{ }^{15}$

The incidence of gestational diabetes has been seen to increase to 7\% higher than expected in the general population. ${ }^{16}$ Older age and PI use were associated with gestational diabetes in a multivariate analysis (odds ratio (OR) 2.3, 95\% confidence interval (CI) 1.0 - 5.3).

Higher rates of hyperglycaemia were seen with nelfinavir (NFV) compared with NVP, $15.6 \%$ v. 8.6\%, ${ }^{17}$ but other cohort cohort reviews conflict. ${ }^{18-20}$ So far data are inconclusive.

\section{FETAL TOXICITY}

The Antiretroviral Pregnancy Registry (APR) looks at potential increases in birth defect prevalence in infants following first-trimester exposure (during which organogenesis occurs) versus second- and third-trimester exposures (see box).

The registry now has sufficient numbers of reports to detect a 2-fold increase in overall anomalies following exposure to $A B C$, atazanavir (ATV), EFV, FTC, indinavir (IDV), lopinavir (LPV), NFV, NVP, ritonavir (RTV), d4T and TDF. For AZT and 3TC there are sufficient numbers of reports to detect a 1.5-fold increase in such anomalies.

The most recent APR report covers the period from 1989 to 31 July 2008, during which time 11950 pregnancies were enrolled. ${ }^{21,22}$ Of these 10471 were evaluable, and $47 \%$ of these mothers were exposed to antiretrovirals

\section{Antiretroviral Pregnancy Registry}

The APR is an international registry, started in 1989, to prospectively monitor potential birth defects in infants exposed to antiretrovirals in utero.

It is one of the largest ongoing pregnancy registries in the world.

The objectives of the registry are to provide early warning of major teratogenicity; estimate the risk of birth defects; and collect supplementary data from animal, clinical and epidemiological studies.

Data collection is through voluntary enrollment by those providing health care to pregnant women exposed to antiretrovirals, and in turn infant followup. The registry has summaries of relevant data for all registered antiretrovirals and reports are updated twice a year.

The majority of reports (88.2\%) are from the USA, with small numbers from elsewhere (e.g. 1\% from South Africa). One of the current goals of the registry is to increase non-US reporting.

Participation in the registry by healthcare providers is encouraged.

http://www.apregistry.com/ 
during the first trimester. A total of 9948 (93\%) live birth outcomes were analysed.

The overall prevalence of defects was 2.7\% (271/9 948), $2.9 \%(126 / 4329)$ in pregnancies with the earliest antiretroviral exposure (in the first trimester) versus 2.6\% (145/ 5 618) for second- and third-trimester exposures.

The APR continues to monitor two drugs that in the past met criteria for evaluation and further monitoring.

AZT was associated with an increased risk of hypospadias among infants in the Women and Infants Transmission Study (WITS). ${ }^{23}$ The WITS data have recently been included in the APR, and when these data are added to the prospective reports, AZT is associated with an increased risk of hypospadias. However, this association is not found when the WITS data are excluded.

In addition a higher rate of congenital anomalies with $\mathrm{ddl}$ has been documented in the APR for several years, but there is no association with any particular defect. However, the overall rate has steadily reduced in recent years and although this last report found a higher than expected 4.4\% (94/3 068) prevalence of defects following first-trimester ddl exposure, it has no apparent pattern and is not statistically significant.

\section{EFAVIRENZ}

EFV has been the antiretroviral that raises the most concern with fetal toxicity in pregnancy.

In pre-clinical trials of EFV, 3/20 cynomolgus macques exposed to the drug during pregnancy produced offspring with significant abnormalities: one with anencephaly and unilateral anophthalmia, one with microphthalmia and one with a cleft palate. As a consequence of these findings EFV was originally classified as FDA category C (Table I). It is notable that EFV is the only antiretroviral that has been studied in this way.

Following four retrospective reports of neural tube defects in infants born to women with first-trimester exposure to EFV, including three cases of meningomyelocele and one Dandy-Walker syndrome, EFV was reclassified as category D in 2005. As these reports were retrospective and therefore the denominator was unknown this reclassification has provoked criticism, since it is not possible to calculate the relative risk. There is also a concern that reporting bias may occur with this drug as the results from the animal studies are well known.

Notably, in the APR report, the prevalence of defects for efavirenz exposures was 3.2\% (13/407), i.e. not elevated above the background population risk, and were no different from first- and second/third-trimester exposure to any other antiretroviral.

\section{OUTCOMES}

Some reports of pregnancy outcomes have shown a significant association between the use of HAART in pregnancy and preterm delivery, but others have not.
TABLE I. US FDA PREGNANCY CATEGORIES FOR CURRENTLY APPROVED ANTIRETROVIRALS

\section{Drug}

FDA category

\section{Abacavir}

Didanosine

Emtricitabine

Lamivudine

Stavudine

Tenofovir

Zalcitabine

Zidovudine

Delavirdine

Efavirenz

Nevirapine

Amprenavir

Atazanavir

Darunavir

Fosamprenavir

Indinavir

Lopinavir +

ritonavir

Nelfinavir

Ritonavir

Saquinavir

Tipranavir

Enfuvirtide

B
B
C
C
B
C
C
C
D
C
C
B
B
C
C
C
B
B
B
B
C
B

FDA categories defined as:

A: Adequate and well-controlled studies of pregnant women fail to demonstrate a risk to the fetus during the first trimester of preg-

nancy (and no evidence exists of risk during later trimesters).

B: Animal reproduction studies fail to demonstrate a risk to the fetus, and adequate but well-controlled studies of pregnant women have not been conducted.

C: Safety in human pregnancy has not been determined; animal studies are either positive for fetal risk or have not been conducted, and the drug should not be used unless the potential benefit outweighs the potential risk to the fetus.

D: Positive evidence of human fetal risk based on adverse reaction data from investigational or marketing experiences, but the potential benefits from the use of the drug among pregnant women might be acceptable despite its potential risks.

$X$ : Animal studies or reports of adverse reactions have indicated that the risk associated with the use of the drug for pregnant women clearly outweighs any possible benefit.

Reports from North America and Western Europe appear to contradict, with greater association observed in European cohorts than in those from the USA, but this is thought to reflect differences in the respective HIVpositive populations and background rates of preterm delivery. ${ }^{24-27}$

Data from African settings are limited. A report from Cote D'Ivoire found in a multivariate analysis of 309 pregnancies that HAART initiated at least 28 weeks before delivery was independently associated with low birth weight (OR 2.53, 95\% Cl $1.20-5.35, p=0.015$ ) compared with a prevention of mother-to-child transmission (PMTCT) regimen. ${ }^{28}$ In this study the investigators were unable to calculate gestational age of the infants.

Of live infants reported in the Kisumu Breastfeeding Study, 17\% were preterm and $13.3 \%$ were of low birth weight.29 This analysis found no difference between NNRTI or PI use: 19\% versus 14\% for those receiving nevirapine and nelfinavir, respectively $(p=0.08)$. It also did not find a difference in the rate of low birth weight: 14\%

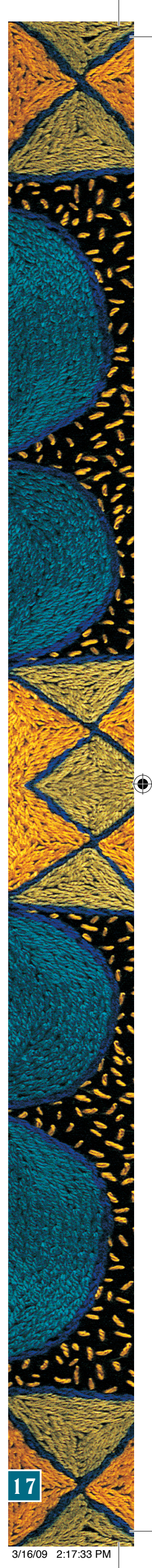


versus $12 \%$ for nevirapine and nelfinavir, respectively $(p=0.7)$. However, the median duration of antenatal exposure in this study was short: 5.1 weeks for nevirapine and 6 weeks for nelfinavir.

In a prospective review of pregnant women in Botswana, HAART continued versus other (AZT, HAART initiated or no ARV intervention) was associated with the infant being small for gestational age (adjusted odds ratio (AOR) $1.8,95 \% \mathrm{Cl} 1.2-2.8)$, as was HAART initiated versus AZT by 30 weeks (AOR 2.8, 95\% Cl $1.4-5.7$ ). ${ }^{30}$ For preterm delivery the AORs for HAART continued versus other and HAART initiated versus AZT were 1.2 (95\% Cl 0.8 - 1.9) and $1.2(95 \% \mathrm{Cl} 0.6-2.7)$, respectively. These associations require further study across all settings.

\section{REFERENCES}

1. de Ruiter A, Mercey D, Anderson J, et al. British HIV Association and Children's HIV Association guidelines for the management of HIV infection in pregnant women 2008. HIV Med 2008; 9: 452-502

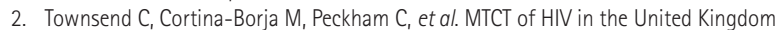
and Ireland, 1990-2004. 14th Conference on Retroviruses and Opportunistic Infections, Los Angeles, CA, 25-28 February 2007. Abstract 761.

3. Townsend $C$, Cortina-Borja M, Peckham $C$, et al. Very low risk of mother-to-child transmission of HIV in women on HAART achieving viral suppression in the UK and Ireland. AIDS 2008: 22: 973-981.

4. Cooper ER, Charurat M, Mofenson LM, et al. Combination antiretroviral strategies for the treatment of pregnant HIV-1-infected women and prevention of perinatal HIV-1 transmission. J Acquir Immune Defic Sydr 2002; 29: 484-494.

5. Black V, Hoffman RM, Sugar CA, et al. Safety and efficacy of initiating highly active antiretroviral therapy in an integrated antenatal and HIV clinic in Johannesburg, South Africa. J Acquir Immune Defic Syndr 2008; 49(3): 276-281.

6. Ekouevi DK, Patrick A. Coffiea PA, et al. Antiretroviral therapy in pregnant women with advanced HIV disease and pregnancy outcomes in Abidjan, Cote d'Ivoire. AIDS 2008; 22(14): 1815-1820

7. Palombi L, Marazzib MC, Voetbergc A, et al. Treatment acceleration program and the experience of the DREAM program in prevention of mother-to-child transmission of HIV. AIDS 2007: 21: suppl 4, S65-S71.

8. US Food and Drug Administration. Important drug warning - Zerit and Videx. [Retyped letter from Bristol-Myers Squibb Company, dated 5 January 2001.] www. fda.gov/medwatch/safety/2001/zeritttvidex_letter.htm 2001 (accessed 26 February 2009).

9. Electronic Medicines Compendium. http://emc.medicines.org.uk/document. aspx?documentld=322 (accessed 26 February 2009).

10. Baylor M, Truffa M, Gibbs N. Hepatic toxicity of antiretrovirals in HIV-infected pregnant women: a review of the FDA's adverse event reporting system. 11th Conference on Retroviruses and Opportunistic Infections, San Francisco, CA, 25-28 February 2004. Abstract 944

11. Thomas $T_{1}$ Amornkul $P_{1}$ Mwidau J, et al. Preliminary findings: incidence of serious adverse events attributed to nevirapine among women enrolled in an ongoing trial using HAART to prevent mother-to-child HIV transmission. 12th Conference on Retroviruses and Opportunistic Infections, Boston, MA, 22-25 February 2005. Abstract 809.
12. Natarajan $U$, Pym A, McDonald $C$, et al. The safety of nevirapine in pregnancy. HIV Med 2007; 8: 64-69

13. Phanupak N, Apornpong T, Limpongsanurak S, et al. Toxicities from nevirapine-based ART regimens in pregnant women with CD4 counts between 250 and 350 cells $\mathrm{mm}^{3}$. 14th Conference on Retroviruses and Opportunistic Infections, Los Angeles, CA, 25-28 February 2007. Abstract 752.

14. Guidelines. Antiretroviral Therapy in Adults: January 2008. Southern African Journal of HIV Medicine 2008; January: 18-31.

15. Justman JE, Benning $L$, Danoff $A$, et al. Protease inhibitor use and the incidence of diabetes mellitus in a large cohort of HIV-infected women. AIDS 2003; 32: 298-302.

16. Gonzalez-Tome MI, Ramos J, Solis I, et al. Gestational diabetes and ART in pregnant HIV-1-infected women. 12th Conference on Retroviruses and Opportunistic Infections, Boston, MA, 22-25 February 2005. Abstract 68.

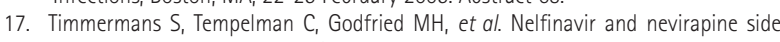
effects during pregnancy. AIDS 2005; 19: 795-799.

18. Dinsmoor MJ, Forrest ST. Lack of an effect of protease inhibitor use on glucose tolerance during pregnancy. Infect Dis Obstet Gynecol 2002; 10: 187-191.

19. Tang JH, Sheffield JS, Grimes J, et al. Effect of protease inhibitor therapy on glucose intolerance in pregnancy. Obstet Gynecol 2006; 107: 1115-1119.

20. Hitti J, Andersen J, McComsey G, et al. Protease inhibitor based antiretroviral therapy and glucose tolerance in pregnancy: AIDS Clinical Trials Group A5084. Am J Obstet Gynecol 2007; 196: 331

21. Beckerman KP, Covington $D$, Dominguez $K$, et al. Antiretroviral Pregnancy Registry (APR) at 10,000 prospective reports. 9th International Congress on Drug Therapy in HIV Infection, Glasgow, 9-13 November 2008. Abstract 0223. http://www. jiasociety.org/content/11/S1/022 (accessed 26 February 2009).

22. Antiretroviral Pregnancy Registry International Interim Report for 1 January 1989 through 31 January 2008. Wilmington, NC: Registry Coordinating Center, 2008. http://www.APRegistry.com (accessed 26 February 2009).

23. Watts DH, Li D, Handelsman E, et al. Assessment of birth defects according to maternal therapy among infants in the Women and Infants Transmission Study. J Acquir Immune Defic Syndr 2007; 44(3): 299-305.

24. European Collaborative Study. Increased risk of adverse pregnancy outcomes in HIV-infected women treated with highly active antiretroviral therapy in Europe. AIDS 2004; 18: 2337-2339.

25. Townsend CL, Cortina-Borja M, Peckham CS, Tookey PA. Antiretroviral therapy and premature delivery in diagnosed HIV-infected women in the United Kingdom and Ireland. AIDS 2007; 21: 1019-1026

26. Schulte J, Dominguez K, Sukalac T, Bohannon B, Fowler MG, for the Pediatric Spectrum of HIV Disease Consortium. Declines in low birth weight and preterm birth among infants who were born to HIV-infected women during an era of increased use of maternal antiretroviral drugs: pediatric spectrum of HIV disease, 1989-2004. Pediatrics 2007: 119: e900-e906.

27. Townsend $\mathrm{CL}$, Schulte J, Thorne $\mathrm{C}$, et al. Differences in the association between HAART in pregnancy and premature delivery: a comparison of three studies in the United States and Europe. 17th International AIDS Conference, 3-8 August 2008, Mexico City. Abstract MOPE0498.

28. Koumavi Ekouevi D, Tonwe-Gold B, Becquet $R$, et al. Low birth weight with nevirapine-based ART in Abidjan, Côte d'lvoire: The ANRS Ditrame Plus Cohort and MTCT-Plus Initiative, 2001 to 2007. 15th Conference on Retroviruses and Opportunistic Infections, Boston, 3-6 February 2008. Abstract 641.

29. Masaba R, Ndivo R, Nyangau I, et al. Comparison of adverse foetal outcomes in HIV-1-infected antiretroviral-naïve pregnant women who have received combivir and either nevirapine or nelfinavir for prevention of mother-to-child transmission antenatally. 15th Conference on Retroviruses and Opportunistic Infections, Boston, 3-6 February 2008. Abstract 640.

30. Chen J, Ribaudo H, Ogwu A, et al. Risk factors for adverse pregnancy outcomes among HIV-infected women in Gaborone, Botswana. 16th Conference on Retroviruses and Opportunistic Infections, Montreal, 8-11 February 2009. Abstract 949.

A man co-infected with HIV and MDR-TB receives attention from an occupational therapist at home. He has been left paralysed due to HIV-related complications and relies on his aunt - in her 60s - to care for him. She is too weak to move him on her own, and with two other cases of TB in the household struggles to care for everyone and maintain the house. During this project the NGO Yizane Sakhe assisted in finding a new wheelchair for him.

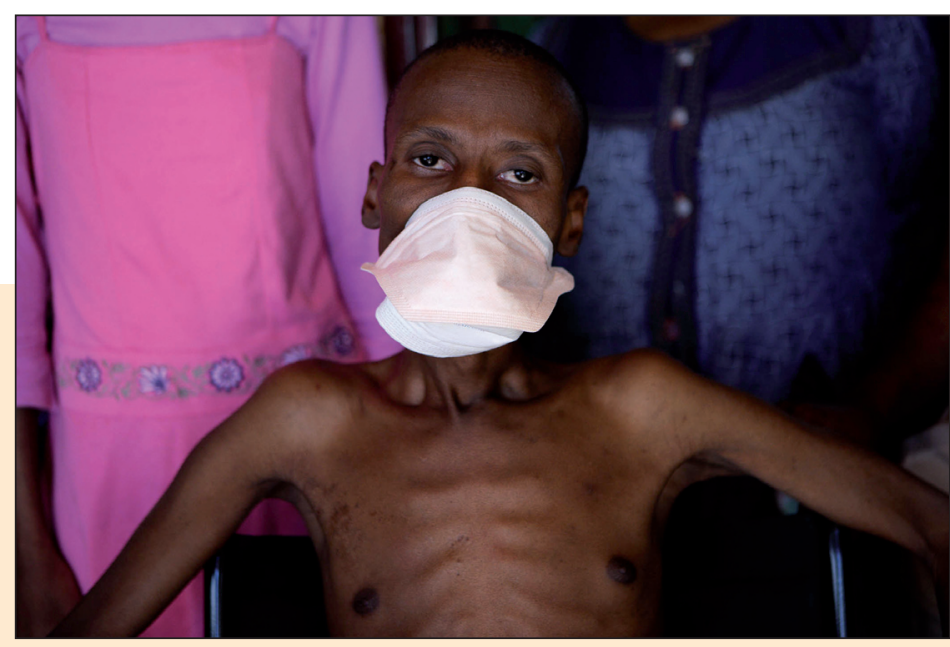

